\title{
Synchronization of the Stochastic Fitzhugh-Nagumo Equations to Periodic Forcing $(*)$.
}

\author{
A. LONGTIN \\ Département de Physique, Université d'Ottawa \\ 150 Louis Pasteur, Ottawa, Ontario, Canada K1N 6N5
}

(ricevuto il 24 Aprile 1995)

\begin{abstract}
Summary. - The Fitzhugh-Nagumo equations (FHN) provide a simple description of the dynamics of a large class of neurons. We characterize synchronization and stochastic resonance in this model using two complementary points of view: the signal-to-noise ratio (SNR), and the absolute as well as normalized peak heights of the interspike interval histograms (ISIHs). At low stimulus frequencies, multiple firings can occur during one period, while at high frequencies, the refractoriness precludes firing at every cycle. The behaviors of the SNR and ISIHs are thus investigated at low, medium and high frequencies to illustrate special synchronization properties of the FHN system. In particular, the behavior of the SNR vs. noise is found to be similar for forcing amplitudes just below and above that at which a $2: 1$ deterministic phase-locked firing solution becomes stable. Our results rely on an accurate method of estimation of the power spectrum of the point process formed by the firing times. A theoretical analysis for the shape of the simulated power spectra is also presented.
\end{abstract}

PACS 87.10 - General, theoretical, and mathematical biophysics (including logic of biosystems, quantum biology, and relevant aspects of thermodynamics, information theory, cybernetics, and bionics).

PACS 05.40 - Fluctuation phenomena, random processes, and Brownian motion.

PACS 02.50 - Probability theory, stochastic processes, and statistics.

PACS 01.30.Cc - Conference proceedings.

\section{1. - Introduction.}

This paper describes various aspects of the synchronization of an excitable neuron [1] to an external stimulus in the presence of stochastic forcing. An excitable neuron can be described by variables that vary on fast time scales, such as the transmembrane voltage, as well as by other fast or slow variables (sect. 3).

(*) Paper presented at the International Workshop «Fluctuations in Physics and Biology: Stochastic Resonance, Signal Processing and Related Phenomena», Elba, 5-10 June 1994. 

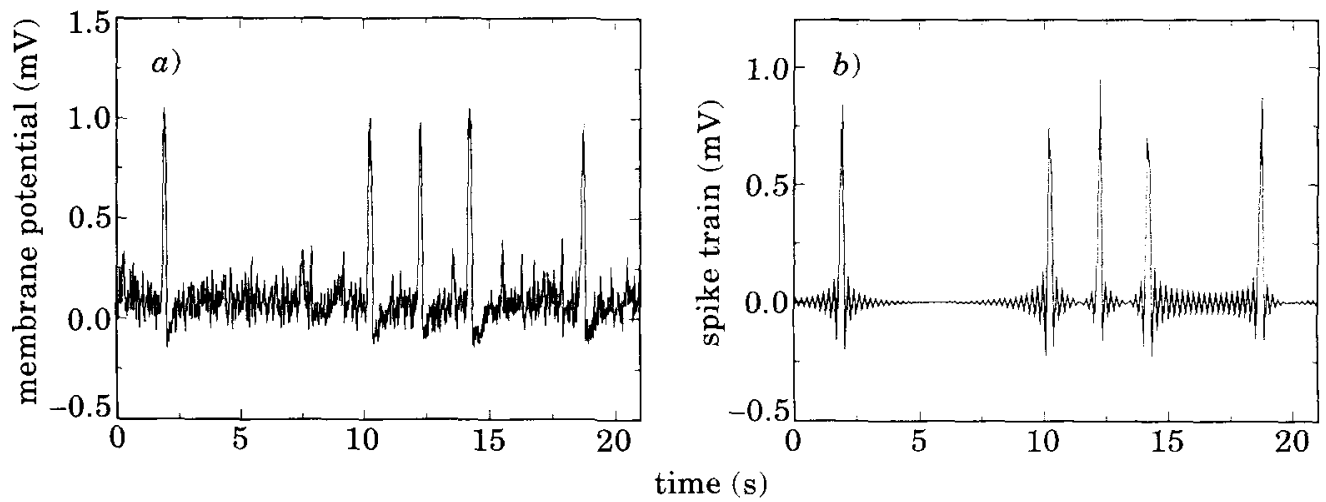

Fig. 1. - Membrane voltage (a)) and corresponding spike trains (b)) from numerical simulations of eq. (5). A delta-spike occurs when the potential crosses the threshold $\theta$ from below. These spikes are then convolved $(b))$ with $\sin \left(2 \pi f_{\mathrm{s}} t\right) /\left(2 \pi f_{\mathrm{s}} t\right)$ to avoid aliasing and produce a flat spectral window up to $f_{\mathrm{s}}[2]$. Parameters are $a=0.5, b=0.12, d=1.0, r=0.03, \beta=3.75, \varepsilon=0.005$, $D=9 \cdot 10^{-6}, t_{\mathrm{c}}=0.01, f_{\mathrm{s}}=4.55$. The integration time step is 0.005 , and the spiking threshold is $\theta=0.5$.

Excitability is characterized by a single globally attracting fixed point known as the resting potential or $V_{0}$. Under stochastic forces due to various ionic events, the voltage executes a random walk around this resting state. If this random walk reaches a certain threshold value $\theta$, the voltage executes a rapid positive excursion, followed by a rapid repolarization back to the negative resting voltage (fig. 1a)). Compared to the subthreshold motion around $V_{0}$ and $\theta$, the shape of this "action potential» or «spike» is perturbed only slightly by noise. It is generally accepted that the information communicated to post-synaptic neurons is contained uniquely in the time of occurrence of these spikes [3].

During each cycle of a periodic stimulus, the voltage first approaches, then recedes from the threshold, in turn raising then lowering the firing probability. The case of interest in this paper is that in which the stimulus alone is too small to induce firing: noise becomes essential to transduce information about the stimulus into a spike train. "Skipping» [4] can then arise if the cell fires in phase with the stimulus, but not at every cycle of the stimulus. However, sect. 6 shows that the condition of «no deterministic firing» is not necessary for skipping to appear: a stochastically perturbed limit cycle oscillation can also, under certain conditions, produce skipping. In the context where no deterministic firing occurs, stochastic resonance (SR) can occur, as was first demonstrated in numerical studies in [5] and [6], and experimentally in [7].

The present study brings together the spectral and residence-time-histogram approaches to study synchronization and SR in excitable neurons. Section 2 describes a few measures of synchronization used in neurophysiology. It also gives a theoretical understanding of the power spectra shapes obtained throughout this paper. Section 3 presents the model studied here, the method used to compute alias-free power spectra with a flat spectral window, and other details of the numerical simulations. Sections 4, 5 and 6 look at various properties of this model under, respectively, low- , medium- and high-frequency conditions. In the latter case, a comparison is made to the deterministic firing case which exhibits a stochastic-resonance-like effect. SR 
from the ISIH point of view is compared to that using spectral methods in sect. 7 and the paper concludes in sect. 8.

\section{2. - Measures of neural synchronization.}

There are various measures of neural synchronization in the neurophysiological literature. Excellent reviews can be found in $[3,8]$. One simple measure, the interspike interval histogram or ISIH, is the equivalent of the residence time histogram previously used to study SR in bistable systems [9,4]. ISIHs (shown in fig. 4) have sharp peaks when periodicities and/or phase locking are present. The temporal ordering of the interspike intervals (ISI) is not reflected in this measure. Nevertheless, spectral analyses of ISIHs are routinely used to derive a measure of synchrony between stimulus and firing, known as «synchronization index», when the frequency is much greater than the zero-stimulus mean firing rate. This index can also be computed by Fourier-transforming a temporal representation of the probability of firing known as the post-stimulus-time histogram. A review of these methods along with recent developments can be found in [10]. Other measures of synchronization based on cycle histograms (an ISIH covering only one stimulus cycle) are described in French et al. [11].

Spectral properties can of course be calculated directly from the point process describing the spike train. In fact, SR has been recently demonstrated in a modulated

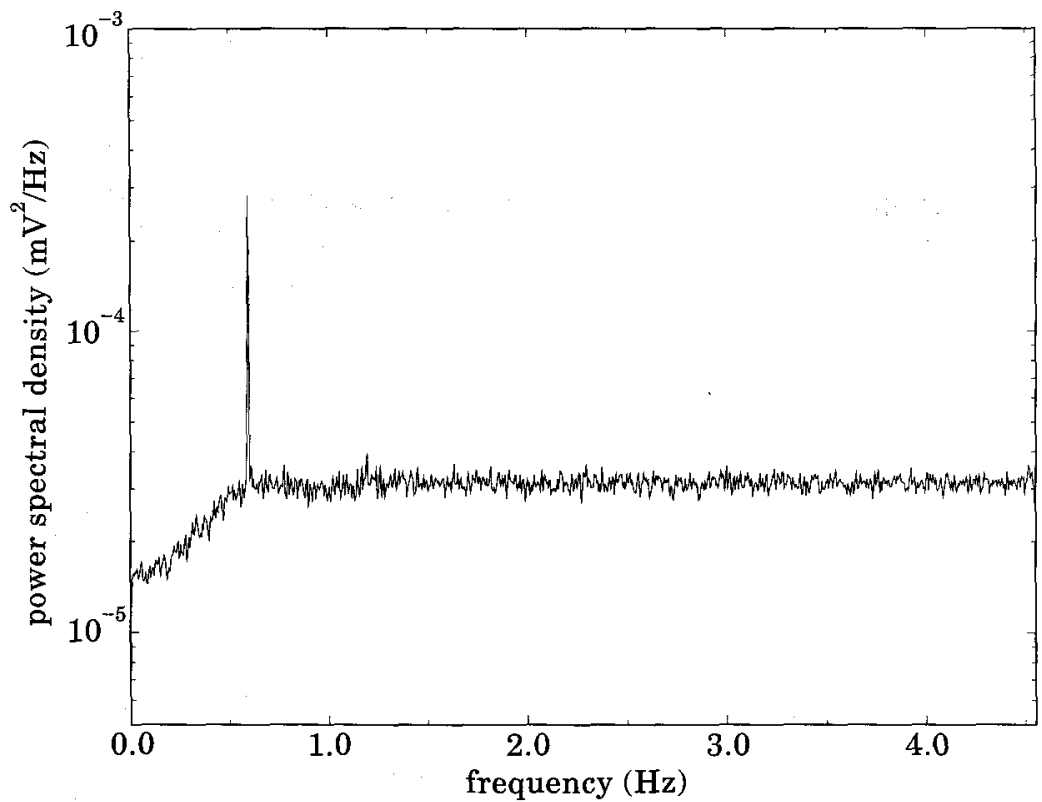

Fig. 2. - Power spectral density obtained from spike trains constructed as in fig. $1 b$ ) from 250 realizations of the stochastic process in eq. (5). Parameters are as in fig. 1. Each realization consists of $10^{5}$ time steps, of which the first $10^{4}$ were discarded as transients. Note the flat spectrum out to the cut-off frequency $f_{\mathrm{s}}=4.55$, characteristic of a Poisson process. 
point process in [12]. A periodic train of action potentials can be modelled by a Dirac comb

$$
x(t)=\sum_{m=-\infty}^{\infty} \delta\left(t-m T_{0}\right),
$$

where $T_{0}$ is the period. It is well known that the power spectrum of $x(t)$ is a set of delta-functions at integer multiples of $f_{0}=1 / T_{0}$ :

$$
S_{x}(\omega)=\frac{2 \pi}{T_{0}^{2}} \sum_{n=-\infty}^{\infty} \delta\left(\omega-\frac{2 \pi n}{T_{0}}\right) .
$$

The spectrum for the case where the ISIs are equal to a constant plus a small random perturbation has been studied in the literature (see, e.g.,[13]). This analysis is useful to understand many but not all features of power spectra under skipping conditions. In fact, our simulations of the FHN system below reveal that increasing the noise
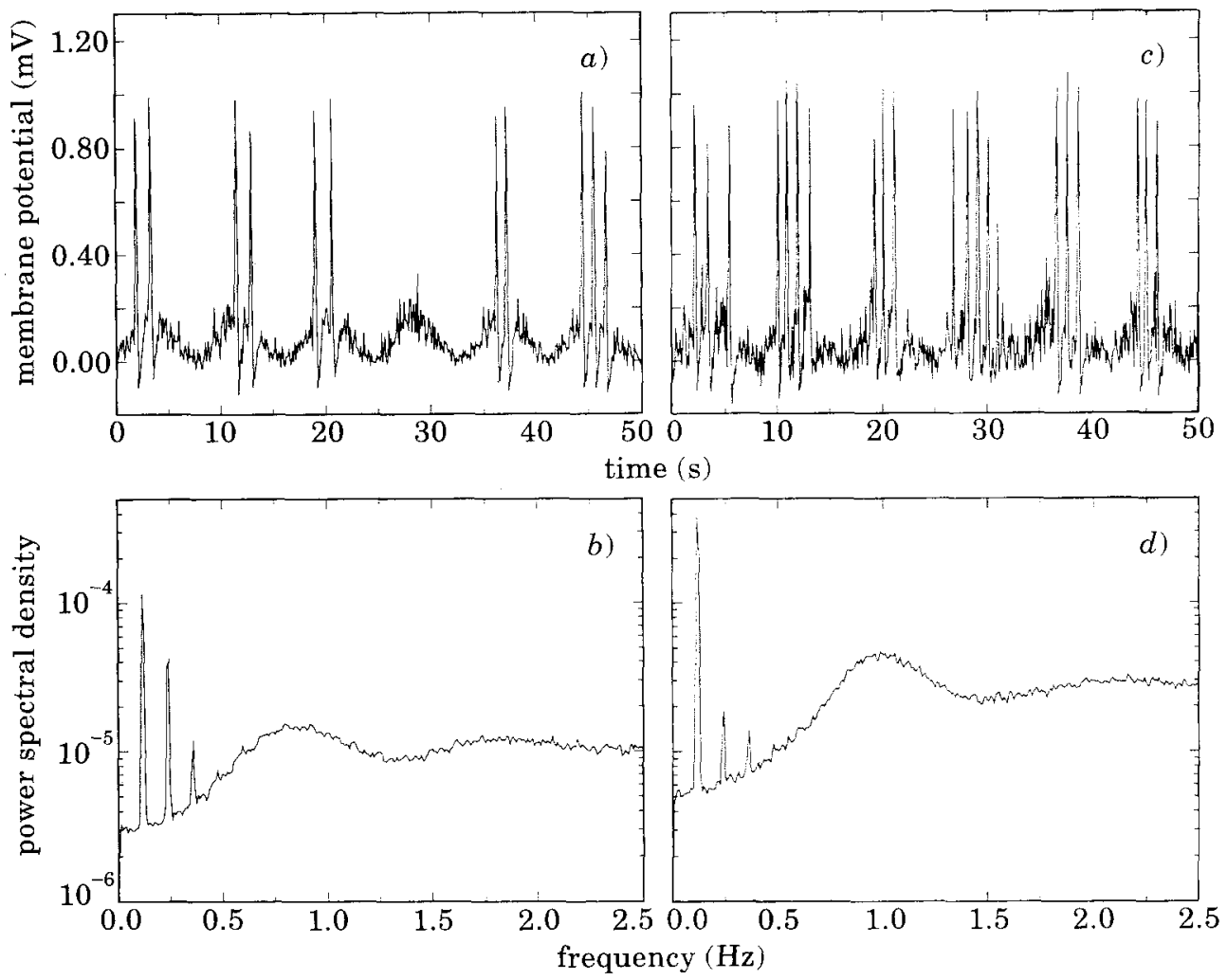

Fig. 3. - Voltage time series from eq. (5) and spike train power spectra for low-frequency conditions: $\beta=0.75\left(f_{0}=0.119\right)$. The noise intensity is $D=2.5 \cdot 10^{-6}$ for panels $\left.a\right)$, $\left.b\right)$, and $D=10^{-5}$ for panels $c$ ), $d$ ). The multiple firings near the stimulus maximum contribute to the high signal-to-noise ratios (see fig. 5). Other parameters are $a=0.5, b=0.12, d=1.0, r=0.1$, $\varepsilon=0.005, t_{\mathrm{c}}=0.01, f_{\mathrm{s}}=8.0, \theta=0.5$. Integration time step is 0.005 . Spectra were computed by averaging 250 realizations of 61200 time steps, for each of which the first 10000 time steps were discarded as transients. 

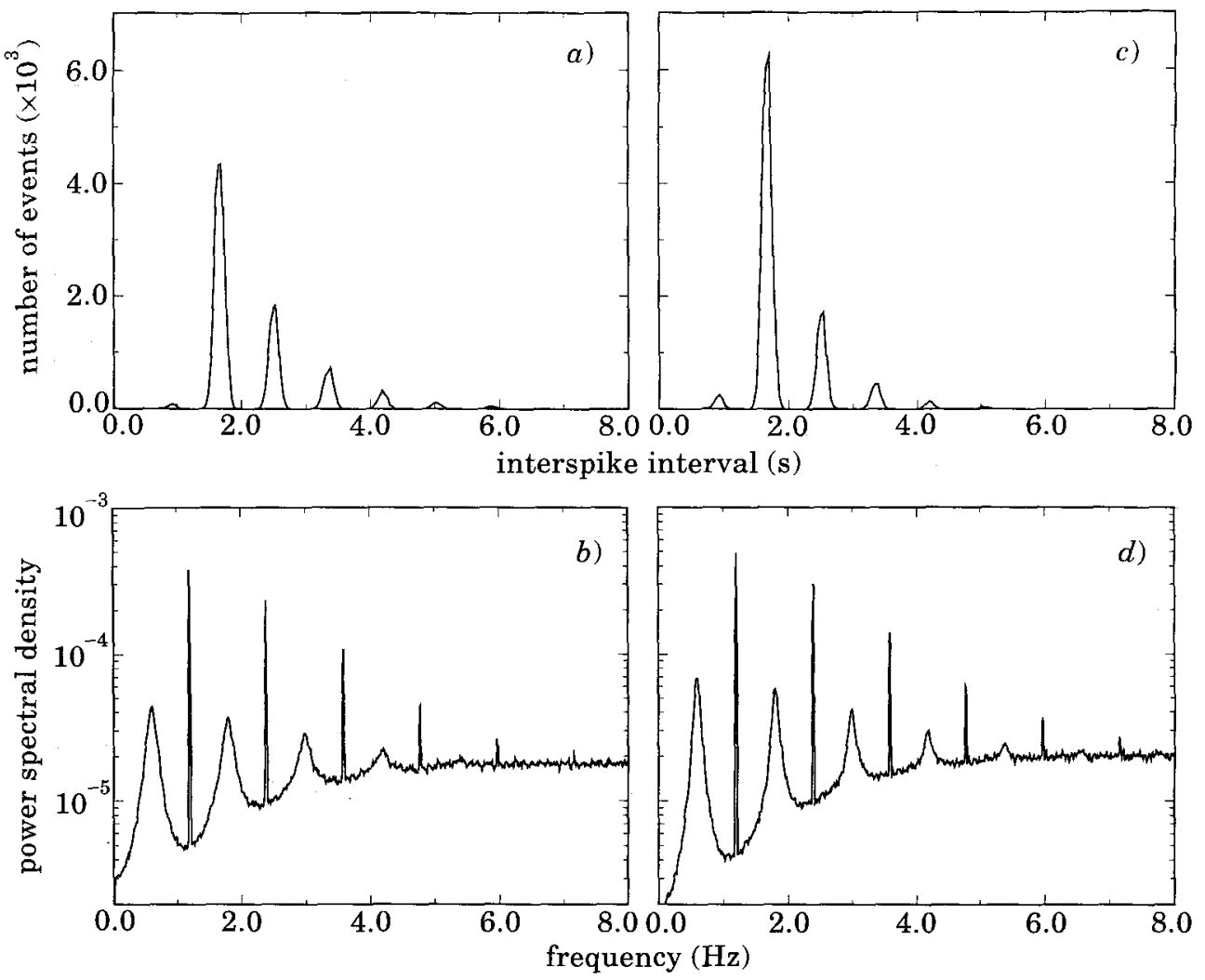

Fig. 4. - Interspike interval histograms and spike train power spectra for eq. (5) under highfrequency $(\beta=7.5)$ and large-amplitude conditions. In $a), b), r=0.20$, there is no deterministic firing, while in $c$ ), $d$ ), $r=0.22$, there is deterministic firing every other cycle of the stimulus (2:1 phase locking). Other parameters are $a=0.5, b=0.12, d=1.0, \varepsilon=0.005, D=2.5 \cdot 10^{-6}$, $t_{\mathrm{c}}=0.01, f_{\mathrm{s}}=12.36$. Integration time step is 0.005 . Spectra are obtained from 500 realizations of 43134 steps discarding the first 10000 steps for each one.

intensity $D$ causes less stimulus cycles to be skipped. When SR occurs, the first peak of the ISIH at $T_{0}$ is clearly the highest, and the signal resembles a periodic signal with jitter, even though a bit of skipping occurs. Assume the $n$-th spike occurs at time $t_{n}=$ $=n T_{0}+\xi_{n}$, where the $\xi_{n}$ are independent random perturbations with distribution $\phi(\xi)$. The ISIH is then a Gaussian centered on $T_{0}$. The power spectrum is given by [13]

$$
S_{x}(\omega)=\frac{1}{T_{0}}\left[1-|\Phi(\omega)|^{2}+\frac{2 \pi}{T_{0}}|\Phi(\omega)|^{2} \sum_{n=-\infty}^{\infty} \delta\left(\omega-\frac{2 \pi n}{T_{0}}\right)\right],
$$

where $\Phi(\omega)$ is the Fourier transform of the probability density $\phi(\xi)$. We will discuss this result in the context where the $\xi$ are Gaussian-distributed with zero mean and standard deviation $\sigma$, as the peaks in the ISIHs computed below (see also [5]) are approximately Gaussian. The characteristic function then takes the form $\Phi(\omega)=$ $=\exp \left[-\left(\omega^{2} \sigma^{2}\right) / 2\right]$. The first two terms in eq. (3) characterize the jitter statistics. They produce a noise background which increases sigmoidally, leveling off at the value 
$T_{0}^{-1}$. The last term is a discrete periodic spectrum which accounts for the underlying periodicity. The discrete components are weighted by the term $|\Phi(\omega)|^{2}$. For example, a typical Gaussian noise causes the amplitude of the components to decrease with increasing frequency, rather than remain constant as in eq. (2).

The spectra presented in this paper at low (fig. 3), medium (fig. 2) and high (fig. 4) stimulus frequency all present discrete components at integer multiples of the driving frequency, with their amplitude decreasing with increasing frequency. The sigmoidal increase in $S(\omega)$ due to "noisy periodicity" is also seen, but closer analysis reveals that its precise shape is governed more by the noise-induced limit cycle (a special property of the FHN system: see sect. 3) than by the stimulus (except at high frequencies: see sect. 6). It is possible to extend the above calculation to compute a signal-to-noise ratio (SNR) based on eq. (3). For Gaussian noise, we find

$$
\operatorname{SNR}(\mathrm{dB})=10 \log \left[\frac{\omega_{0} \exp \left[-\omega_{0}^{2} \sigma^{2}\right]}{1-\exp \left[-\omega_{0}^{2} \sigma^{2}\right]}\right] .
$$

In the context of SR, the behavior of this SNR as a function of $\sigma$ is of interest. The SNR monotonically decreases from $\infty$ as $\sigma \rightarrow 0$ to $-\infty$ as $\sigma \rightarrow \infty$. Thus, with noisy periodicity, increasing noise simply deteriorates synchronization to the stimulus. So while this heuristic analysis helps in the understanding of the spectra, it must be expanded to include skipping in order to see SR; this will be presented elsewhere.

\section{3. - Simulations of the Fitzhugh-Nagumo equations.}

Our study of stochastic synchronization focusses on the Fitzhugh-Nagumo model in the excitable regime with periodic forcing on the recovery variable $[1,5]$

$$
\left\{\begin{array}{l}
\varepsilon \frac{\mathrm{d} v}{\mathrm{~d} t}=v(v-a)(1-v)-w+\eta(t), \\
\frac{\mathrm{d} w}{\mathrm{~d} t}=v-\mathrm{d} w-(b+r \sin \beta t), \\
\frac{\mathrm{d} \eta}{\mathrm{d} t}=-\lambda \eta+\lambda \xi(t),
\end{array}\right.
$$

where $\langle\xi(t)\rangle=0$ and $\langle\xi(t) \xi(s)\rangle=2 D \delta(t-s)$. We chose the exponentially correlated Ornstein-Uhlenbeck process $\eta(t)$ for the noise; its intensity is $D \lambda$, and its correlation time $t_{\mathrm{c}}=\lambda^{-1}$ was set to 0.01 , a time scale comparable to that of the fast variable (the precise value of $t_{\mathrm{c}}$ will depend on the particular neuron under study). The power spectrum of $\eta(t)$ is flat up to the cut-off frequency $\lambda$. These equations are integrated as in [5].

In the absence of periodic and stochastic forcing, this system can fire periodically if $b>b_{\mathrm{H}}=0.264$ (Hopf bifurcation). At $b_{\mathrm{H}}$, the firing period is 1.14, and it decreases slowly as $b$ increases, reaching, e.g., 0.86 when $b=0.30$. All our simulations are done for $b=0.12$. While this is quite far from $b_{\mathrm{H}}$, the limit cycle is nevertheless «induced" by the noise, i.e. the system spends some time near this solution which exists only for neighboring parameters when $D=0$. And its presence is manifest in all our spectra as 
a bump near $f=0.9$, which is some average value of the limit cycle frequencies for $b>b_{\mathrm{H}}$. When $r>0$, the threshold for periodic firing depends on $r$ and $\beta$ [14]. A largeamplitude stimulus highlights this limit cycle «bump» (see fig. 4) as it (along with the noise) effectively modulates $b$ and periodically brings it close to or beyond $b_{\mathrm{H}}$. This bump is also accompanied by its own harmonics.

A spike is said to occur when $x(t)$ crosses the threshold value $\theta=0.5$ from below. Our results depend quantitatively rather than qualitatively on the value of $\theta$, provided it is not too close to $V_{0}$ (resting potential) nor to the peak of the action potential when $D=0$ (for the small $t_{\mathrm{c}}$ chosen here, fluctuations near this maximum will occur for large $D$ ). Further, a crossing is counted only if it occurred more than $\approx$ $\approx 0.4$ (the refractory period) after the previous spike.

31. Power spectral density. - Care must then be taken to obtain a good estimate of the power spectral density $S(f)$ of the point process formed by the spikes over the frequency range of interest. An excellent method has been proposed in [2]. It avoids aliasing and produces a flat spectral window for frequencies up to $f_{\mathrm{s}}$, the frequency at which the spike train is sampled (see, e.g., fig. 2). It requires that the spikes be convolved with the function $\sin \left(2 \pi f_{\mathrm{s}} t\right) /\left(2 \pi f_{\mathrm{s}} t\right)$ (fig. 1b)). The 4096 point power spectra and 200 bin ISIHs are constructed from many different realizations of the stochastic process equation (5). The mean was subtracted from the sampled spike train, followed by a Hanning-type windowing of the data. The signal strength, here measured at the signal frequency $f_{0}$ only, is calculated by summing five bins centered on $f_{0}$. The noise floor is estimated from the average of three points to the right and three to the left of these five bins. The signal-to-noise ratio is calculated as

$$
\mathrm{SNR}=10 \log \frac{\int_{\Delta} S\left(f_{0}\right) d f}{N\left(f_{0}\right)},
$$

where $\Delta$ stands for the five «signal» bins. We have found that our results are qualitatively similar (with lower SNR values) if only the maximum $S\left(f_{0}\right)$ is taken. Our SNR differs slightly from other definitions (see, e.g., [7]) since it uses the total area under the signal peak. All our results are qualitatively similar (e.g., SR occurs at the same value of $D$ ) if the area of the peak above the noise floor is used (a factor of five must be subtracted from the ratio in eq. (6) before taking the logarithm). The statistical error in estimating the SNR is smaller than the size of the symbols used to plot our data. Further, the spectra are not phase-randomized since the spike train for each realization is sampled (after a fixed time for transients to decay) starting at the same phase of the external stimulus.

In the following sections we look at the behavior of this SNR and of ISIHs under special frequency and amplitude conditions chosen to illustrate special synchronization properties of the FHN system. A general comment on the shape of the spectra can be made at this point, as it pertains to the noise-induced limit cycle. Except for fig. 4, the spectra obtained below show the limit cycle bump and its harmonics even when the stimulus has zero amplitude (data not shown). Putting $r=0$ simply eliminates the sharp signal peaks. The increase to the first bump presumably does not occur by the same mechanism as that which gives rise to the sigmoidal increase in eq. (3). In fact, in fig. 2 and 3 , the bump along with the trough preceeding it become more pronounced as either $D$ or $b$ increase, two effects which make the limit cycle 
more prominent. The trough occurs because low frequencies can induce limit cycle firing in the frequency range of the bump. In other words, low-frequency fluctuations are filtered out by the limit cycle which acts as a resonator. However, we suspect the attenuation of the harmonics of the bump occurs by a mechanism similar to that discussed in sect. 2.

\section{4. - Synchronization at low frequency.}

We first consider the case $\beta=0.75$ and $r=0.1$. For this low frequency, deterministic firing occurs when $r>0.173$. A stimulus frequency which is low compared to the internal relaxation time of the neuron will initiate zero, one or many spikes per stimulus cycle. The same is true if low noise is also present, as is shown in fig. 3. At higher noise (fig. 3c)), many spikes are seen during each cycle, and the instantaneous firing rate follows the stimulus wave form, leading to high SNRs (see below). This phenomenon is known to occur in mammalian auditory fibers at low frequencies [15]. It is also responsible for the linearization by noise, studied by French et al. [11], of the input-output relation of certain receptor neurons, which is otherwise nonlinear due to rectification and phase locking.

The power spectra in fig. $3 b$ ), $d$ ) consist of sharp spikes at integer multiples of the driving frequency with a decaying envelope. This is a signature of noisy periodicity (sect. 2). This discrete spectrum is superimposed on a noisy background modulated by broad peaks at integer multiples of the noise-induced limit cycle frequency (around $0.9 \mathrm{~Hz}$, sect. 3). These broad peaks are caused by the multiple firings on this limit cycle. They are enhanced by increasing $b$ towards $b_{\mathrm{H}}$. They are also enhanced by the noise (compare fig. $3 b$ ), d)), and especially low-frequency noise which allows the dynamics to spend more time near the limit cycle (thus the trough at low frequency). When the amplitude of the low-frequency stimulus is zero, the sharp peaks disappear, and the bumps are less pronounced, since the noise by itself can cause only brief excursions to the limit cycle. When $D$ is at the intermediate value of $\approx 7.5 \cdot 10^{-6}$, the SNR reaches it maximum, as is shown in fig. 5a). In this case (not shown), many spikes are triggered during the time when the stimulus is near its maximum. As a

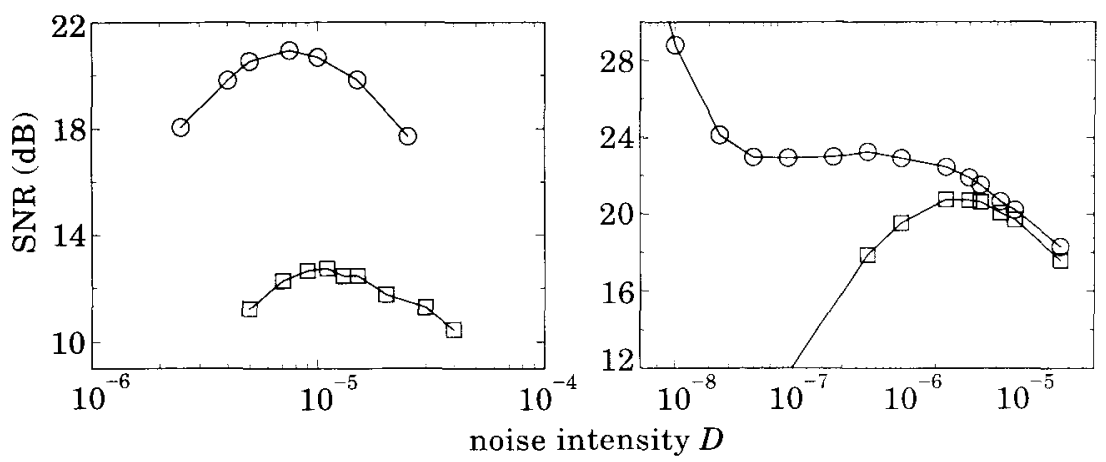

Fig. 5. - Stochastic resonance for (left panel) low- (circles: $\beta=0.75, r=0.1$ ), mid- (squares: $\beta=3.75, r=0.03$ ) and (right panel) high-frequency forcing (squares: $\beta=7.5, r=0.20$; circles: $\beta=7.5$ and $r=0.22$ ). The signal-to-noise ratio (eq. (6)) is plotted $v s$. $D$. Deterministic firing occurs for the $r=0.22$ case. Simulation parameters for $\beta=3.75$ are as in fig. 1 ; those for $\beta=0.75$ are as in fig. 3 , and those for $\beta=7.5$ are as in fig. 4 . 
result, the ISIH peak normally at $T_{0}$ moves to the left, and a sharp peak due to the multiple firings appears at shorter ISIs.

\section{5. - Synchronization at medium frequency.}

Higher frequencies such as the one $(\beta=3.75)$ used in this section tend to produce one-to-one phase locking without multiple firings. The small amplitude $r=0.03$ case (far from the deterministic firing regime) is investigated here. A typical solution and power spectrum were shown in fig. 1 and 2 . This spectrum is for a noise value just below the one at which SR occurs. Signal magnitude and SNR are smaller in this case than in the previous (and next) case, and the modulating amplitude is hardly visible as it is buried in the noise (fig. 1a)). In fact, the second harmonic of the fundamental at $f_{0}$ barely rises out of the noise floor. The SNR is plotted vs. $D$ in fig. $5 a$ ); it is lower because the signal is small. However, the noise does not seem to affect the shape of the spikes as much in this case as in the low- and high-frequency cases (not shown). The relations between the SNR and the ISIH for this case are discussed in sect. 7 below. The initial increase in $S(\omega)$ is hardly affected by the presence of the weak stimulus (not shown). It is due again to the noise-induced limit cycle around $0.9 \mathrm{~Hz}$ which is visible but small, and to jitter (sect. 2). It becomes important when $D>1.5 \cdot 10^{-5}$ (not shown). This is in contrast to the cases in sect. 4 and 6 since (sect. 3) here the small stimulus (plus noise) does not bring $b$ near to $b_{\mathrm{H}}$ as often.

\section{6. - Synchronization at high frequency.}

Due to refractoriness, a neuron is incapable of firing at every cycle of a superthreshold stimulus of high frequency. The combination of noise and refractoriness can easily produce skipping, regardless of whether or not deterministic stimulus-induced firing occurs. This is shown in fig. 4, where deterministic firing occurs when $r>0.215$. Panels $a$ ), $b$ ) for $r=0.20$ are qualitatively very similar to panels $c$ ), $d$ ) for $r=0.22$. This is due to the fact that 2:1 phase locking, which exists for $D=0$ and $r=0.22$, is induced by the noise when $r=0.20$. When $D=0$ and $r=0.22$, all the peaks in the spectrum are of the same width and strength (data not shown). The sharp peaks for $D>0$ are at integer multiples of the forcing frequency $(1.19 \mathrm{~Hz})$, and at even multiples of the first broad peak corresponding to the frequency of the 2:1 phaselocked solution $(0.598 \mathrm{~Hz})$. The presence of noise produces a decreasing envelope of both series of peaks (sect. 2). Further, the rate of increase in $S(\omega)$ is proportional to $D$ as in eq. (3).

It is intriguing that the sharpest and highest peak occurs at $f_{0}=1.19$, even though the ISIH has very few events corresponding to this frequency. In fact, the most probable ISI corresponds to $2 T_{0}$, not $T_{0}$. An intuitive reason for this effect is that each ISI at $n T_{0}(n \neq 1)$ has one of its corresponding harmonics at $f_{0}$, leading to a high power at $f_{0}$. Another insight comes from considering each ISI as the sum of two random variables: an integer Poisson variable multiplied by $T_{0}$, and a Gaussian variable. By putting a dead-time (mimicking refractoriness) which excludes events at $T_{0}$, we found that the ISIH for a sequence of such ISIs can be made identical to those in fig. 4. The associated power spectra were also identical to those observed here. A more rigorous analysis will be carried out elsewhere. 
The subthreshold case $r=0.20$ exhibits SR (fig. 5). Moreover, as the $r=0.20$ and $r=0.22$ cases are barely distinguishable at the noise levels of fig. 4, one might ask whether the superthreshold dynamics display behavior similar to SR. The behavior of the SNR vs. $D$ for $r=0.22$ is plotted above that for $r=0.20$. It is seen that this SNR diverges as $D \rightarrow 0$ since the limiting motion is periodic (sect. 2). There is a range however where this SNR plateaus, and even increases slightly, before resuming its downward trend. This SR-like effect, which is a form of noise-induced order, most likely arises for the same reason that normal SR does: a commensurate relation between the rate at which firings would occur with $r=0$ and the rate induced by the stimulus.

\section{7. - Normalized and unnormalized ISIHs.}

Stochastic resonance has also been investigated from the residence-timehistogram point of view [9]. A similar resonant behavior of the ISIH peaks was demonstrated in models of excitable neurons in [5] and [6]. Here we take a closer look at this problem by comparing SR from the spectral and ISIH points of view. We have performed two measures on the ISIH at different values of $D: 1$ ) the number and 2 ) the normalized probability of ISIs falling near the maximum of the first two peaks of the ISIH. The histogram contains 200 bins ranging from 0 to $8 T_{0}$. The number of events «near the maximum» is defined as six bins centered on $T_{0}$ (or $2 T_{0}$ for the second peak). The probability is obtained by dividing this number by the total number of events in the ISIH. Figures $6 a$ ) and $b$ ) plot, respectively, this probability and number of events as a function of $D$. The upper curves (circles) are for the high-frequency high-amplitude case, while the lower curves (squares) are for the medium-frequency low-amplitude case. As in fig. 5, all curves start at small values, but only the behavior near the "resonance» is plotted. Further, only the numbers for the second peak P2 at $2 T_{0}$ are plotted as it is always well defined and its behavior
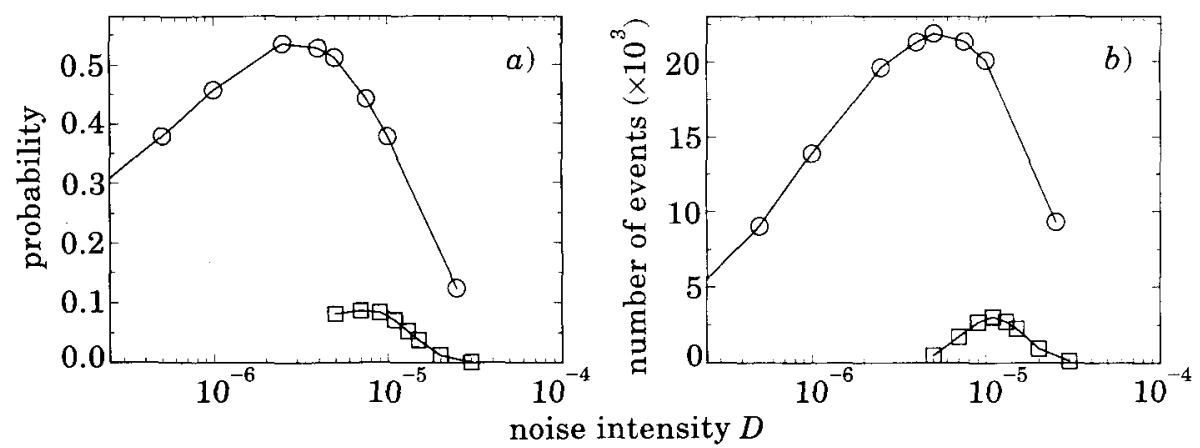

Fig. 6. - Behavior of the integrated second peak (P2) of the ISIH as a function of D. a) The normalized probability is used. $b$ ) The absolute number of events is used. Circles are for high frequency $\beta=7.5$ and $r=0.2$; squares are for $\beta=3.75$ and low amplitude $r=0.03$. The probabilities go through their maximum at a smaller value of $D$ than the absolute numbers of events. The results are obtained from the same simulations as those used to generate fig. 5 . 
correlates well with the spectral SNR. In contrast, the first peak shifts towards lower ISIs and $D$ increases, and is eventually engulfed by the gamma-type noise distribution, concurring with results in [9].

A comparison of fig. $6 a$ ) and $b$ ) shows that the probability curves reach their maximum at slightly smaller values of $D$ than do the unnormalized curves. Another observation arises from the comparison of these curves to the SNR curves in fig. 5. At high frequency, the probability and the SNR peak near the same value of $D$, while the maximum number of events occurs at a larger $D$. In contrast, for $\beta=3.75$ and low amplitude, the maximum number of events and the SNR reach their maximum near the same $D$. However in this latter case $r$ is small, and the ISIH is not as smooth as in the high-frequency case, and longer simulations may be needed before drawing a final conclusion. Thus there does not seem to be one fixed rule by which these various indices can be connected. For now, the only conclusion is that SR from the ISIH point of view occurs at slightly lower noise values, as was the case in a recent experimental study [7]. The behavior of P2 is similar to that described in the adiabatic limit by [9] for the bistable system, although such comparisons should be done with caution since our simulations are done at quite high frequency and the noise is slightly colored.

\section{8. - Conclusion.}

Our study has described synchronization of the FHN excitable system to different stimuli. Our results rely on accurate alias-free and spectrally flat estimates of power spectra of spike trains generated by the FHN system. We have found that SR occurs over a wide range of frequencies and amplitudes, and have compared the SNR and ISIH points of view. From the neurobiological point of view, it is not clear whether SNR, probability or absolute numbers of spikes is more significant. For example, a P2 event might be more probable at $D_{1}$ than at $D_{2}>D_{1}$, yet in absolute numbers, there are less $\mathrm{P} 2$ events at $D_{1}$ than at $D_{2}$. The resolution of this problem will probably depend on the particular neural system under study. Perhaps numbers of spikes are not so important, but accurate input-output phase relationships are. And neural circuits which use noise to sharpen phase relationships, e.g., through SR, may thereby increase their timing and computational capabilities.

This work was supported by NSERC Canada and by NIMH (USA) grant R01 MH47184-01. The author wishes to thank F. Moss for helpful discussions.

\section{REFERENCES}

[1] Alexander J. C., Doedel E. J. and Othmer H. G., SIAM (Soc. Industr. Appl. Math.) J. Appl. Math., 50 (1990) 1373.

[2] French A. S. and Holden A. V., Kybernetik, 8 (1971) 165.

[3] Moore G. P., Perkel D. H. and Segundo J. P., Ann. Rev. Physiol., 28 (1966) 493.

[4] Longtin A., Bulsara A., Pierson D. and Moss F., Biol. Cyberm., 70 (1994) 569.

[5] Longtin A., J. Stat. Phys., 70 (1993) 309.

[6] Chialvo D. R. and Apkarian V., J. Stat. Phys., 70 (1993) 375. 
[7] Douglass J. K., Wilkens L., Pantazelou E. and Moss F., Nature, 365 (1993) 337.

[8] MCA. SAYERS B., Infering significance from biological signals, in Biomedical Engineering Systems, edited by M. Clynes and J. Milsum (McGraw-Hill, New-York) 1970, p. 84.

[9] Zhou T., Moss F. and Jung P., Phys. Rev. A, 42 (1990) 3161.

[10] Gummer A. W., Hearing Res., 55 (1991) 143.

[11] French A. S., Holden A. V. and Stein R. B., Kybernetik, 11 (1972) 15.

[12] Wiesenfeld K., Pierson D., Pantazelou E. and Moss F., Phys. Rev. Lett., 72 (1994) 2125.

[13] Coggshall J. C., Kybemetik, 13 (1973) 30.

[14] Hochmair-Desoyer I. J., Hochmair E. S., Motz H. and RatTay F., Neuroscience, 13 (1984) 553.

[15] Motz H. and RatTay F., Neuroscience, 18 (1986) 699. 